\title{
Gastric Volvulus Secondary to Paraesophageal Hernia Causing Gastric Necrosis and Perforation of the Gastroesophageal Junction
}

\author{
Nasir Saleemª, b, Nizar Senussia ${ }^{\mathrm{a}}$, Muhammad Farhan ${ }^{\mathrm{a}}$
}

\begin{abstract}
Gastric volvulus is a potentially lethal condition with significant morbidity and mortality if not recognized and managed promptly and appropriately. We report a case of gastric volvulus secondary to paraesophageal hernia causing gastric necrosis and perforation of the gastroesophageal junction. An 82-year-old woman with a large paraesophageal hernia was admitted to our hospital with vague epigastric and right-sided chest pain. She was taken to surgery and laparotomy findings included a large paraesophageal hernia, gastric volvulus with ischemia, frank gangrene and perforation at the gastroesophageal junction. She underwent reduction of the hernia and gastric volvulus with partial gastroesophagectomy and formation of an esophagogastrostomy anastomosis. Due to the patient's old age and associated co-morbidities, her prognosis remained poor post-operatively and she was transferred to hospice care as per family wishes. This case report is intended to educate clinicians to maintain a high index of suspicion for acute gastric volvulus and its potential complications in the appropriate clinical setting to allow for early surgical intervention and appropriate management.
\end{abstract}

Keywords: Gastroesophageal hernia; Gastric volvulus; Gastric necrosis

\section{Introduction}

Gastric volvulus is characterized by rotation of the stomach along its long or short axis leading to variable degrees of gastric outlet obstruction, which may present acutely or chronically. Gastric volvulus is rare. The incidence peaks after the fifth decade with adults constituting $80-90 \%$ of cases. No association with sex or race has been reported. One of the more

Manuscript accepted for publication May 20, 2015

aDepartment of Internal Medicine, Presence St. Joseph Hospital, Chicago, IL, USA

${ }^{b}$ Corresponding Author: Nasir Saleem, Department of Internal Medicine, Presence St. Joseph Hospital, 2900 N Lake Shore Dr., Chicago, IL 60657, USA. Email: nasirsaleemmd@gmail.com

doi: http://dx.doi.org/10.14740/jmc2191w important risk factors for gastric volvulus is paraesophageal hernia, others being age, other diaphragmatic abnormalities including sliding hiatal hernia, phrenic nerve paralysis, and anatomic gastrointestinal abnormalities. Rotation of the stomach more than $180^{\circ}$ can cause gastric strangulation leading to ischemia and potentially necrosis, perforation, and sepsis. Morbidity and mortality related to acute gastric volvulus is high if unrecognized, underscoring the need for early diagnosis and treatment. The possibility of gastric volvulus with hiatal hernia must be recognized.

\section{Case Report}

An 82-year-old white female, with past medical history of hypertension, atrial fibrillation, congestive heart failure, and chronic kidney disease, presented to the emergency department with a 12-h history of upper abdominal and right-sided chest pain, poorly localized to the epigastrium and right upper quadrant, $5 / 10$ in severity, deep in nature and radiating to her back. No aggravating or alleviating factors were noted. The patient reported mild shortness of breath and dizziness but denied nausea, vomiting, diarrhea, constipation, dysphagia, hematemesis, hematochezia, or melena, and her last bowel movement was 1 day prior to presentation. Her abdominal pain started 2 weeks ago, was waxing and waning over this time and became constant and more severe $12 \mathrm{~h}$ prior to presentation. She also had a history of heartburn but stated that this pain felt different than heartburn and was not relieved by antacids. On examination in the emergency department her blood pressure was $133 / 92$, pulse 102 and irregular, respiratory rate $18 / \mathrm{min}$ and was maintaining oxygen saturation to $94 \%$ on room air. Lung auscultation revealed decreased breath sounds on the right side but physical examination was otherwise unremarkable. A 12lead EKG showed atrial fibrillation with a rapid ventricular response and no ischemic changes. She was given intravenous metoprolol and her ventricular rate slowed down. Acute coronary syndrome was ruled out with serial EKGs and troponin. The rest of laboratory workup revealed a WBC of 11.9 with $83 \%$ segmented neutrophils, amylase of 79 and lipase of 66 . An initial serum lactic acid was 4.2 with a subsequent rise to 4.8 after $4 \mathrm{~h}$. A portable chest X-ray revealed a large hiatal hernia and a large right pleural effusion (Fig. 1). An initial $\mathrm{CT}$ abdomen without contrast revealed a loculated right pleu- 


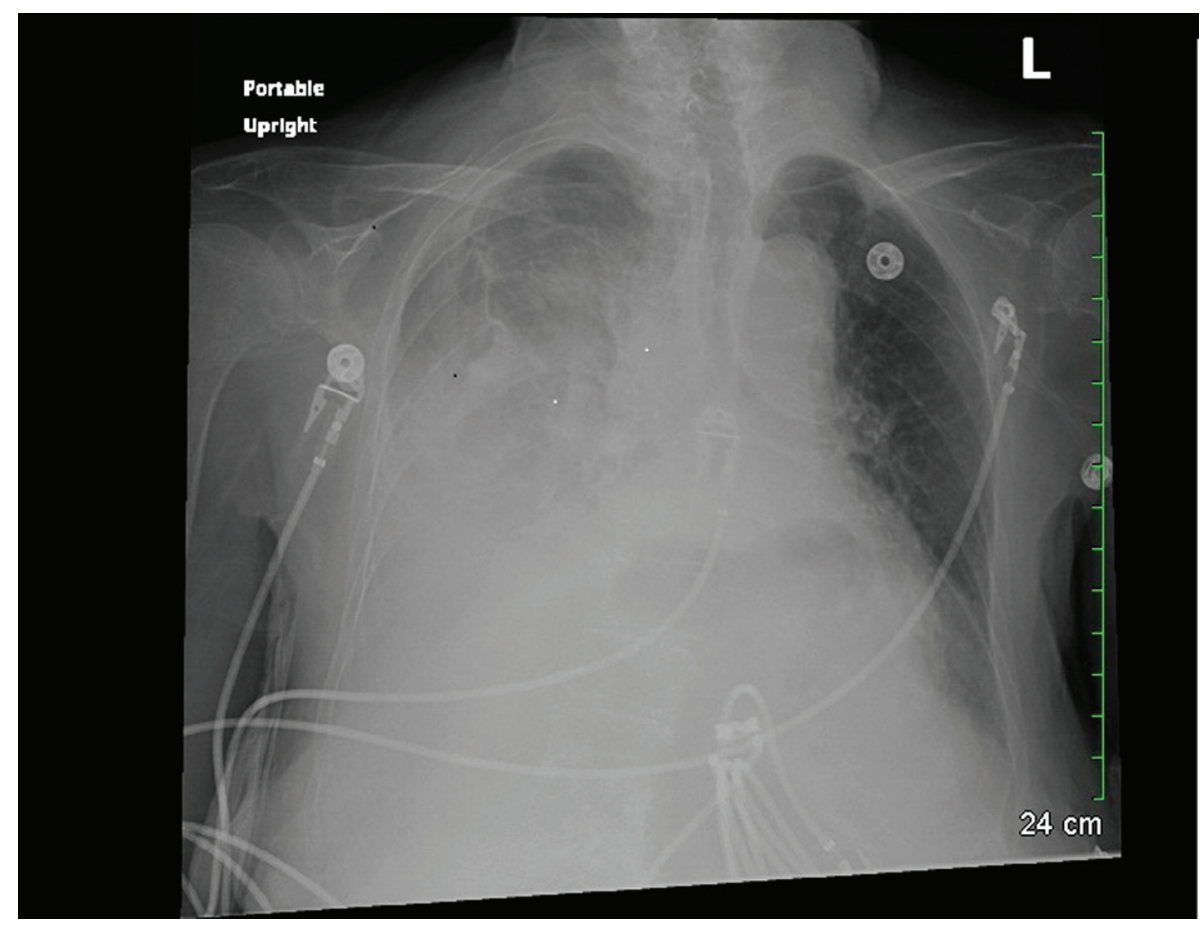

Figure 1. Portable chest $X$-ray showing a large hiatal hernia with superimposed right-sided opacification indicating a large pleural effusion.

ral effusion, a large diaphragmatic hernia with penetration of practically the entire stomach into the thoracic cavity and an epigastric mass presumed to be hematoma. No extraluminal gas or fluid was found and there was no evidence of acute pancreatitis. A subsequent contrast enhanced CT scan of the chest was reported as abnormal, depicting progressive nonspecific changes in the right hemithorax characterized as a large complicated effusion, presumably hemorrhagic or inflammatory, and a $12 \mathrm{~cm}$ right lower intrapleural non-homogeneous hyperdensity, with likely possibilities of hematoma, extension from the esophagogastric junction, occult perforation, volvulus or malignancy. No obvious acute pathology of the abdomen or pelvis was reported (Fig. 2, 3). Owing to the patient's increasing discomfort and pain, CT scan findings, a rising white cell count and lactic acid, along with the lack of a definitive infective source and/or diagnosis, the patient was transferred to the intensive care unit for closer monitoring and care. By this stage she was already started on intravenous fluids and empiric antibiotics. The surgical on-call team was consulted and with a high index of clinical suspicion for gastric volvulus along with the right lower intrapleural hyperdensity, it was deemed appropriate to take the patient to surgery following detailed discussion regarding the potential risks and benefits with the family. The laparotomy findings included a type 3 paraesophageal hernia, gastric volvulus with ischemia, frank gangrene and perforation at the gastroesophageal junction. The procedure performed included reduction of the hernia and gastric volvulus with partial gastroesophagectomy and formation of an esophagogastrostomy anastomosis. Post surgical findings described the perforation as a large defect with a sac of inflammatory tissue encasing multiple food products that appeared to be seeds or grapes. This area was adequately debrided. Further findings included a large amount of dark blood and bilious material below the diaphragmatic crura. A subsequent right posterolateral thoracotomy revealed additional debris in the chest that was cleaned up. Although the patient tolerated the procedure well, her condition remained critical. The patient remained intubated and was transferred to the intensive care unit. Subsequently her condition deteriorated as she developed sepsis and multi-organ failure. She was maintained on intravenous fluids, pressors and broad spectrum antibiotics, and later transferred to hospice care as per family wishes.

\section{Discussion}

Gastric volvulus involves rotation of the stomach along its long or short axis. It is an uncommon entity but can present as a surgical emergency requiring prompt management as the risk of mortality is high [1].

The etiology of gastric volvulus can be classified into primary or secondary to an underlying cause. Primary gastric volvulus occurs due to laxity of the ligaments supporting the stomach, thereby allowing the stomach to twist along its mesentery [2]. Secondary volvuli can occur due to a number of causes including abdominal adhesions, diaphragmatic eventration, splenic abnormalities, phrenic nerve palsy, traumatic diaphragmatic rupture or an intrinsic gastric pathology, like tumors, acting as a lead point for gastric volvulus [2, 3]. A study in 2000 found that the most common predisposing factor is a paraesophageal hiatal hernia (28/36 cases) [4].

Gastric volvulus can be further classified as acute or 


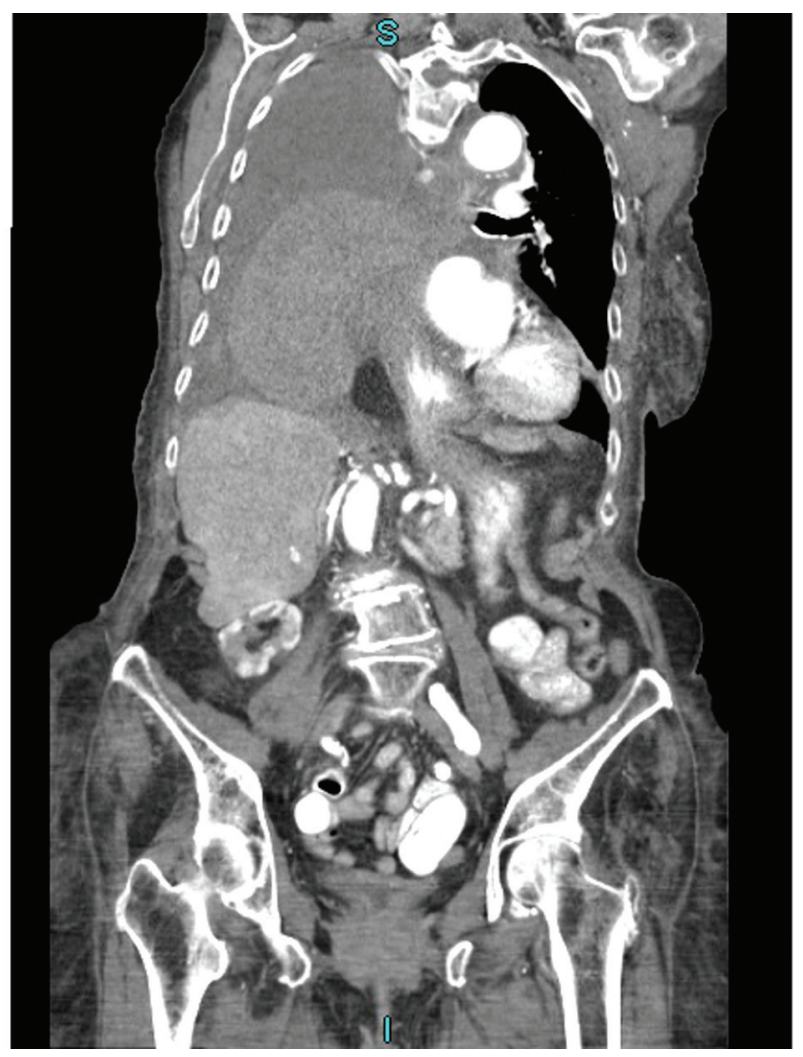

Figure 2. CT chest (coronal section) showing right-sided pleural effusion, a right lower intrapleural inhomogeneous hyperdensity (reported as hematoma extending from the esophagogastric junction vs. volvulus vs. occult perforation) and a large hiatal hernia.

chronic. In the chronic form a partial volvulus occurs which is less likely to cause ischemic injury; however, in the acute form of gastric volvulus the rotation of the stomach is more than $180^{\circ}$ [5]. This can result in a closed-loop obstruction, progressive dilatation of the stomach and eventual ischemia. If not recognized early, this can cause infarction and necrosis of the stomach, resulting in rupture and peritonitis. The mortality associated with such severe complications is approximately 42$56 \%$ [2]. However, it is important to note that the stomach has a rich blood supply; therefore severe ischemic events occur in approximately $5-28 \%$ of patients [3].

Gastric volvulus in $70 \%$ of cases presents in its classical form as the Bortchardt's triad, that is intractable retching, sudden epigastric pain and an inability to pass a nasogastric tube into the stomach [2]. Other presentations include dyspepsia, epigastric pain, dysphagia or chest pain mimicking acute coronary syndrome [6].

The diagnosis of gastric volvulus is often delayed because of the non-specific nature of presenting symptoms [7]. Plain radiographic findings include retrocardiac air, in keeping with the intrathoracic portion of the stomach. Air fluid levels in the mediastinum may also be noted. A barium study may be helpful in establishing the diagnosis and the type of volvulus. It may also determine the degree of obstruction by determining if there is flow of oral contrast into the duodenum [5]. However, in the acute setting, these diagnostic modalities may not

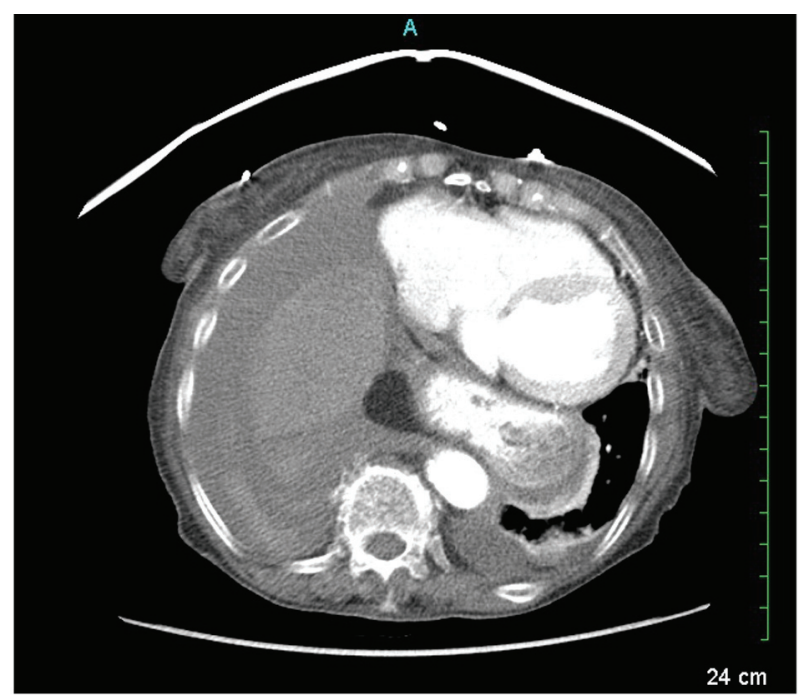

Figure 3. CT chest (axial section) showing right-sided pleural effusion, a right lower intrapleural inhomogeneous hyperdensity (reported as hematoma extending from the esophagogastric junction vs. volvulus vs. occult perforation) and a large hiatal hernia.

be feasible, especially if the patient is critically ill. CT is now commonly performed, helping to delineate the anatomy and to establish any complications arising from a complete volvulus [8], as CT is both highly sensitive and specific for diagnosing acute gastric volvulus [9]. However, it is pertinent to mention that preoperative diagnosis of gastric volvulus is often difficult, and its management involves surgical correction of the pathology followed by institution of resuscitative treatment [10].

Acute gastric volvulus is a rare surgical emergency presenting with atypical symptoms, but one that needs to be recognized and managed early given the high mortality associated with its complications. A high degree of suspicion and early cross-sectional imaging studies are crucial in establishing the diagnosis [1]. It is also important to recognize that patients with paraesophageal or sliding hiatal hernias are at an increased risk of developing gastric volvulus and potential necrosis in the appropriate clinical setting. And although a large hiatal hernia is usually a chronic disorder, surgical treatment is recommended, considering the risk of serious complications [11]. In our patient, the insidious onset and paucity of gastrointestinal symptoms, along with the nonspecific findings on CT abdomen, precluded a very early diagnosis. However, it further illustrates the relevance of gastric volvulus as a possible differential diagnosis in the appropriate clinical setting.

\section{Conflict of Interest}

No.

\section{References}

1. Wijesuriya S, Watura R. Acute gastric volvulus: an un- 
common complication of a hiatus hernia. BMJ Case Rep. 2011;2011

2. Chau B, Dufel S. Gastric volvulus. Emerg Med J. 2007;24(6):446-447.

3. Sevcik WE, Steiner IP. Acute gastric volvulus: case report and review of the literature. CJEM. 1999;1(3):200-203.

4. Teague WJ, Ackroyd R, Watson DI, Devitt PG. Changing patterns in the management of gastric volvulus over 14 years. Br J Surg. 2000;87(3):358-361.

5. Peterson CM, Anderson JS, Hara AK, Carenza JW, Menias CO. Volvulus of the gastrointestinal tract: appearances at multimodality imaging. Radiographics. 2009;29(5):1281-1293.

6. Porter DN, Emerson C, Ward P. The best way to a woman's heart? Emerg Med J. 2011;28(3):247-248.

7. Laurent S, Grayet D, Lavigne Ch M. Acute and chronic gastric volvulus: radical different prognosis and management. Case report. Acta Chir Belg. 2010;110(1):76-79.

8. Singham S, Sounness B. Mesenteroaxial volvulus in an adult: time is of the essence in acute presentation. Biomed Imaging Interv J. 2009;5(3):e18.

9. Millet I, Orliac C, Alili C, Guillon F, Taourel P. Computed tomography findings of acute gastric volvulus. Eur Radiol. 2014;24(12):3115-3122.

10. Altintoprak F, Yalkin O, Dikicier E, Kivilcim T, Arslan Y, Gunduz Y, Ozkan OV. A rare etiology of acute abdominal syndrome in adults: Gastric volvulus - Cases series. Int J Surg Case Rep. 2014;5(10):731-734.

11. Matsumoto N, Oki E, Morita M, Kakeji Y, Egashira A, Sadanaga N, Maehara Y. Successful treatment of acute esophageal necrosis caused by intrathoracic gastric volvulus: report of a case. Surg Today. 2009;39(12):1068-1072. 